\title{
Flowability of Gel-Matrix and Magnetorheological Response for Carrageenan Magnetic Hydrogels
}

\author{
Junko Ikeda, Tomoki Kurihara, Keiju Ogura, Shota Akama, Mika Kawai and Tetsu Mitsumata * (D) \\ Graduate School of Science and Technology, Niigata University, Niigata 950-2181, Japan; \\ f17k501b@mail.cc.niigata-u.ac.jp (J.I.); kuri.tomokies@gmail.com (T.K.); t17g428d@mail.cc.niigata-u.ac.jp (K.O.); \\ aaa.rock@icloud.com (S.A.); mikagoro@eng.niigata-u.ac.jp (M.K.) \\ * Correspondence: tetsu@eng.niigata-u.ac.jp; Tel.: +81-(0)25-262-6884
}

check for

updates

Citation: Ikeda, J.; Kurihara, T.; Ogura, K.; Akama, S.; Kawai, M.; Mitsumata, T. Flowability of Gel-Matrix and Magnetorheological Response for Carrageenan Magnetic Hydrogels. Gels 2021, 7, 56. https:// doi.org/10.3390/gels7020056

Academic Editors: Toshikazu

Takigawa, Takamasa Sakai

and Kenji Urayama

Received: 21 April 2021

Accepted: 2 May 2021

Published: 6 May 2021

Publisher's Note: MDPI stays neutral with regard to jurisdictional claims in published maps and institutional affiliations.

Copyright: (c) 2021 by the authors. Licensee MDPI, Basel, Switzerland. This article is an open access article distributed under the terms and conditions of the Creative Commons Attribution (CC BY) license (https:// creativecommons.org/licenses/by/ $4.0 /)$.
Abstract: The relationship between rheological features in the absence of a magnetic field and magnetic response was investigated for $\mathrm{k}$-carrageenan magnetic hydrogels containing carbonyl iron particles. The concentration of carrageenan was varied from 1.0 to $5.0 \mathrm{wt} \%$, while the concentration of carbonyl iron was kept at $70 \mathrm{wt} \%$. The magnetic response revealed that the change in storage modulus $\Delta G^{\prime}$ decreased inversely proportional to the carrageenan concentration. A characteristic strain $\gamma_{1}$ where $G^{\prime}$ equals to $G^{\prime \prime}$ was seen in a strain range of $10^{-3}$. It was found that $\Delta G^{\prime}$ was inversely proportional to the characteristic stress at $\gamma_{1}$. Another characteristic strain $\gamma_{2}$ where the loss tangent significantly increased was also analyzed. Similar to the behavior of $\gamma_{1}, \Delta G^{\prime}$ was inversely proportional to $\gamma_{2}$. The characteristic stresses at $\gamma_{1}$ and $\gamma_{2}$ were distributed at 80-720 Pa and 40-310 $\mathrm{Pa}$, respectively. It was revealed that a giant magnetorheology higher than $1 \mathrm{MPa}$ can be observed when the characteristic stresses at $\gamma_{1}$ and $\gamma_{2}$ are below approximately $240 \mathrm{~Pa}$ and $110 \mathrm{~Pa}$, respectively.

Keywords: stimuli-responsive material; magnetic gel; viscoelastic property; magnetorheologylogy; carrageenan

\section{Introduction}

Magnetic soft material consisting of polymeric matrix and magnetic particles demonstrates drastic changes in physical properties in response to magnetic fields, and it has great potential in various applications [1-3]. To achieve a high and fast response of the elasticity change, a variety of improvements have been reported [4-9]. Under a gradient magnetic field, magnetic soft material undergoes elongation behavior proportionally to the field gradient [10]. Under a uniform magnetic field, the magnetorheological effect (MR effect) is observed, where the viscoelastic properties vary in response to the magnetic field. The MR effect is basically caused by an anisotropic structure of magnetic particles formed in a matrix of a cross-linked polymer, which is called a chain structure. The structure can be observed by an optical microscopy when the concentration of magnetic particles is low [11]. Gundermann and Odenbach investigated the movement of magnetic particles for a magnetic elastomer with magnetic particles of $2 \mathrm{wt} \%$ using an X-ray computed tomography (CT). They found that the magnetic particles move and form a chain-like structure in the cross-linked matrix [12]. We also found by an X-ray CT with high resolution that magnetic particles make an anisotropic structure under a magnetic field [13]. When forming the chain structure, the polymer network should be destroyed in local owing to the relative movement of magnetic particles. Actually, an indication has been observed by SEM observation that a carrageenan network was stretched by magnetic particles [14]. If the polymer network is easy to deform or elongate, magnetic particles easily align in the direction of magnetic field, resulting in high and fast magnetic response. Of course, the increase in storage modulus by the structuring of magnetic particles is far higher than the decrease 
in the modulus due to the breaking of cross-linking point; therefore, the modulus change being observed is normally positive.

Magnetic hydrogels made of polysaccharides have been widely investigated as a drug carrier [15-22]. On the other hand, they are known for demonstrating a significant MR effect compared with magneto-responsive gels or elastomers made of synthetic polymers [23-26]. That is, the storage modulus for polysaccharide magnetic hydrogels is dramatically increased by a magnetic field even at high off-field modulus. As we reported briefly [24], this is a feature commonly observed in polysaccharide magnetic hydrogels. As a possible reason for this, we presented a hypothesis that there is a special structure enabling the high mobility of magnetic particles in polysaccharide hydrogels. However, the details relating to the special structure for polysaccharide magnetic hydrogels could not be elucidated in the paper. We focused here on the viscoelastic property of carrageenan magnetic hydrogels and investigated the flowability of magnetic particles in carrageenan gel by measuring the dynamic modulus. Polysaccharide hydrogels are formed by a weak physical bond between polysaccharide chains [27]. Therefore, the physical bond should be easily destructed by weak stress and, vice versa, it is easily reconstituted by self-healing [28-30]. In this study, we discuss the relationship between the flowability of the gel-matrix and the MR amplitude (the change in storage modulus).

\section{Results and Discussion}

Figure 1 displays the strain dependence of storage modulus and loss modulus at $0 \mathrm{mT}$ and $500 \mathrm{mT}$ for magnetic hydrogels with various carrageenan concentrations. The storage modulus at a strain of $10^{-4}$ for $1 \mathrm{wt} \%$ carrageenan was $7.4 \times 10^{3} \mathrm{~Pa}$ at $0 \mathrm{mT}$ and $2.2 \times 10^{6} \mathrm{~Pa}$ at $500 \mathrm{mT}$, which was in good agreement with our previous results $[14,23,24]$. At $0 \mathrm{mT}$, a plateau region of $G^{\prime}$ or $G^{\prime \prime}$ was observed at strains below $10^{-3}$ for all magnetic hydrogels showing linear viscoelasticity. At large strains, $G^{\prime}$ decreased remarkably with the strain, indicating that the cross-linking point was destructed by the large strains. The $G^{\prime \prime}$ first increased at a strain around $10^{-2}$, then took a maximum and decreased, which has been classified as type III flow (weak strain overshoot) classified by Hyun et al. [31]. At a strain slightly higher than the maximum, $G^{\prime}$ crossed with $G^{\prime \prime}$, which indicates that the gel matrix changes from solid-like to liquid-like.
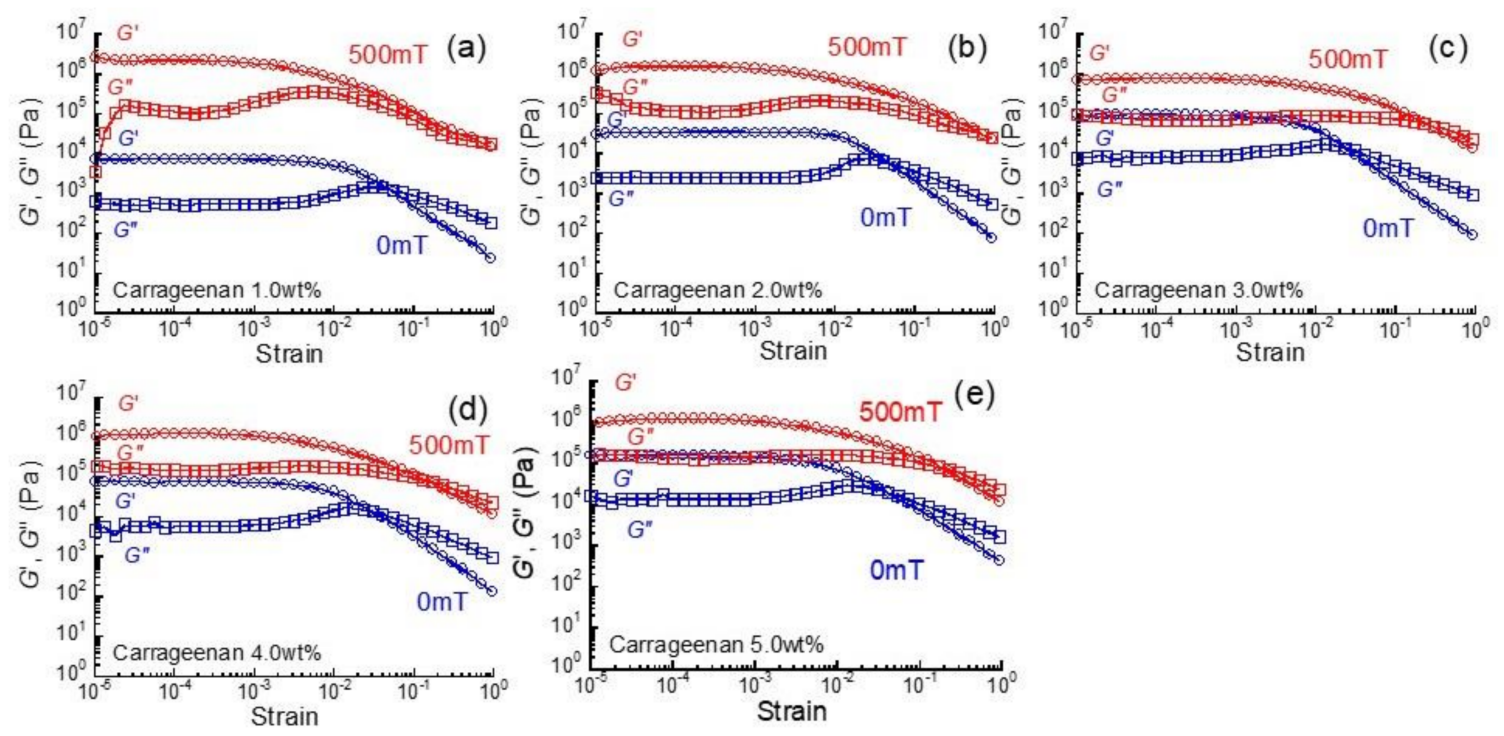

Figure 1. Strain dependence of storage modulus and loss modulus at $0 \mathrm{mT}$ and $500 \mathrm{mT}$ for magnetic hydrogels with various carrageenan concentrations (carbonyl iron (CI): $70 \mathrm{wt} \%, f=1 \mathrm{~Hz}$ ). (a) $1.0 \mathrm{wt} \%$, (b) $2.0 \mathrm{wt} \%$, (c) $3.0 \mathrm{wt} \%$, (d) $4.0 \mathrm{wt} \%$, (e) $5.0 \mathrm{wt} \%$. 
Figure 2a depicts the relationship between the storage modulus and carrageenan concentration for magnetic hydrogels. At $0 \mathrm{mT}$, the storage modulus for magnetic hydrogels took the lowest value at $1.0 \mathrm{wt} \%$, and increased with the carrageenan concentration. The storage modulus was almost constant at concentrations above $3.0 \mathrm{wt} \%$. The storage modulus for carrageenan hydrogels without magnetic particles is also shown in the figure, and it showed a similar trend with magnetic hydrogels. At $500 \mathrm{mT}$, the storage modulus took the maximum value at $1.0 \mathrm{wt} \%$ and gradually decreased with the carrageenan concentration. Similar to the off-field modulus, the on-field modulus was constant at concentrations above $3.0 \mathrm{wt} \%$. It can be considered that a further decrease in the mobility of magnetic particle does not occur at above the concentration.

Figure $2 \mathrm{~b}$ demonstrates the relationship between the change in storage modulus at $500 \mathrm{mT}$ and carrageenan concentration for magnetic hydrogels. Because the on-field modulus is one order of magnitude higher than the off-field modulus, the change in storage modulus $\Delta G^{\prime}$ was inversely proportional to the carrageenan concentration at whole concentrations, as indicated by the dotted line in the figure. It has been widely accepted that the increase in the elastic modulus by magnetic field originates from the chain-like formation of magnetic particles. Actually, the behavior of magnetic particles within matrices of cross-linked polymer has been cleared by $\mu$-CT observation $[12,13]$. It can be considered that the mobility of magnetic particles largely decreased with increasing of the carrageenan concentration owing to a dense network of carrageenan chains.
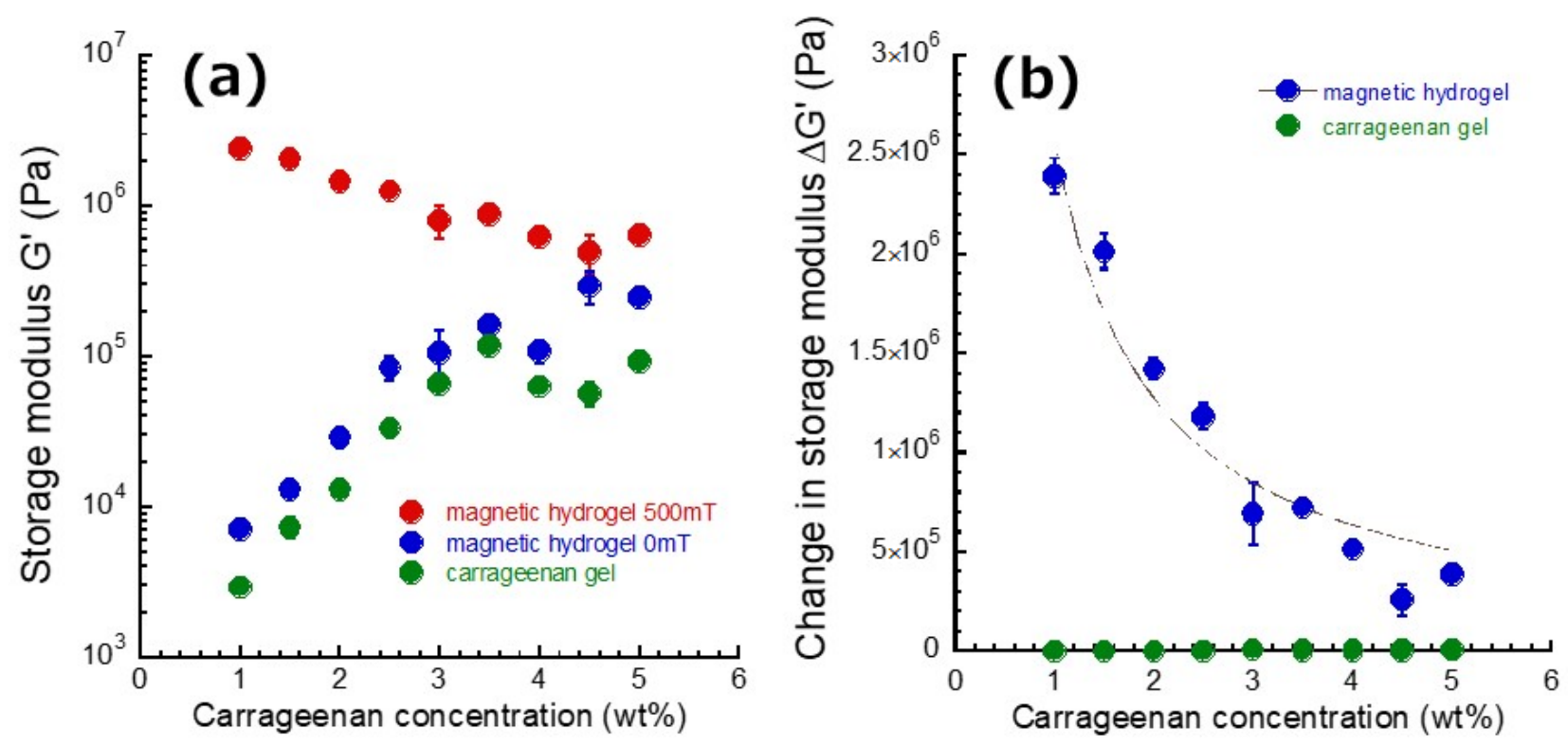

Figure 2. Carrageenan concentration dependence of (a) storage modulus at 0 and $500 \mathrm{mT}$ and (b) change in storage modulus at $500 \mathrm{mT}$ for magnetic hydrogels (CI: $70 \mathrm{wt} \%, f=1 \mathrm{~Hz}$ ) and carrageenan hydrogels without magnetic particles.

Figure 3a shows the relationship between the characteristic strain $\gamma_{1}$ at $0 \mathrm{mT}$ and carrageenan concentration for magnetic hydrogels. The characteristic strain $\gamma_{1}$ was determined by the strain where $G^{\prime}$ equals to $G^{\prime \prime}$ in Figure 1 . The $\gamma_{1}$ for magnetic hydrogels was almost independent of the carrageenan concentration, although it has a trend to decrease with the carrageenan concentration. Interestingly, this means that a physical bond of the carrageenan network becomes easier to break with increasing of the carrageenan concentration. A similar trend of strain softening was also seen in $\gamma_{1}$ for carrageenan hydrogels without magnetic particles. Therefore, this is an intrinsic property for carrageen gel. The absolute values of $\gamma_{1}$ for carrageenan hydrogels without magnetic particles were approximately $1 / 4$ of those for magnetic hydrogels, suggesting that the carrageenan network became tough by incorporating with magnetic particles. 
Figure $3 \mathbf{b}$ demonstrates the relationship between the characteristic strain $\gamma_{2}$ and carrageenan concentration for magnetic hydrogels. Clearly, $\gamma_{2}$ for magnetic hydrogels decreased inversely proportional to the carrageenan concentration. On the other hand, $\gamma_{2}$ for carrageenan hydrogels was almost independent of the carrageenan concentration.

Figure 4 displays the strain dependence of loss tangent for magnetic hydrogels with various carrageenan concentrations. At $0 \mathrm{mT}$, the loss tangent was almost constant at strains below $10^{-3}$ and sharply increased at a certain strain, which is called a characteristic strain $\gamma_{2}$ hereafter. The characteristic strain $\gamma_{2}$ for magnetic hydrogels appeared at strains approximately $10^{-2}$ and clearly decreased with the carrageenan concentration. As $\gamma_{2}$ corresponds to the onset strains where $G^{\prime \prime}$ starts to increase, it can be considered that $\gamma_{2}$ is a characteristic strain at which the physical bond between carrageenan chains and/or the physical contact between magnetic particles through the bound layer of carrageenan start to destruct by the strain. At $500 \mathrm{mT}$, the $\gamma_{2}$ for magnetic hydrogels clearly shifted to lower strains in a range of approximately $10^{-4}$. It can be considered that $\gamma_{2}$ under the magnetic field is the characteristic strain at which the chains of magnetic particles start to be destructed by the strain.
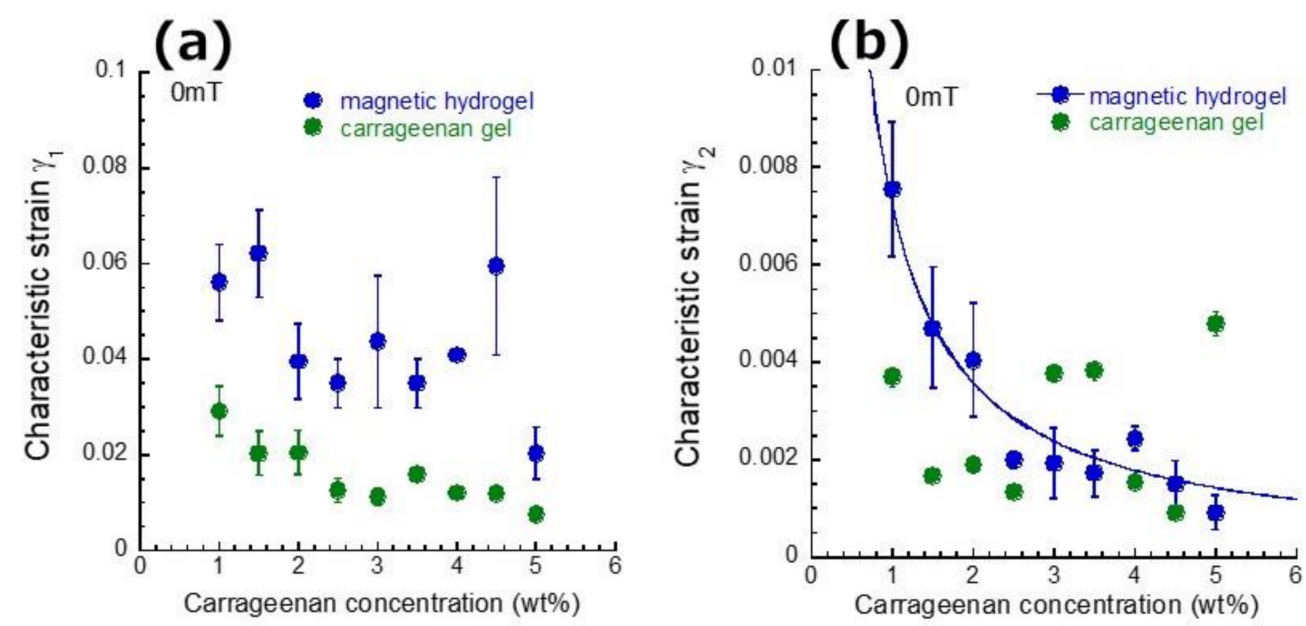

Figure 3. Relationship between characteristic strain (a) $\gamma_{1}$ or $(\mathbf{b}) \gamma_{2}$ and carrageenan concentration for magnetic hydrogels (CI: $70 \mathrm{wt} \%$ ) and carrageenan hydrogels without magnetic particles.
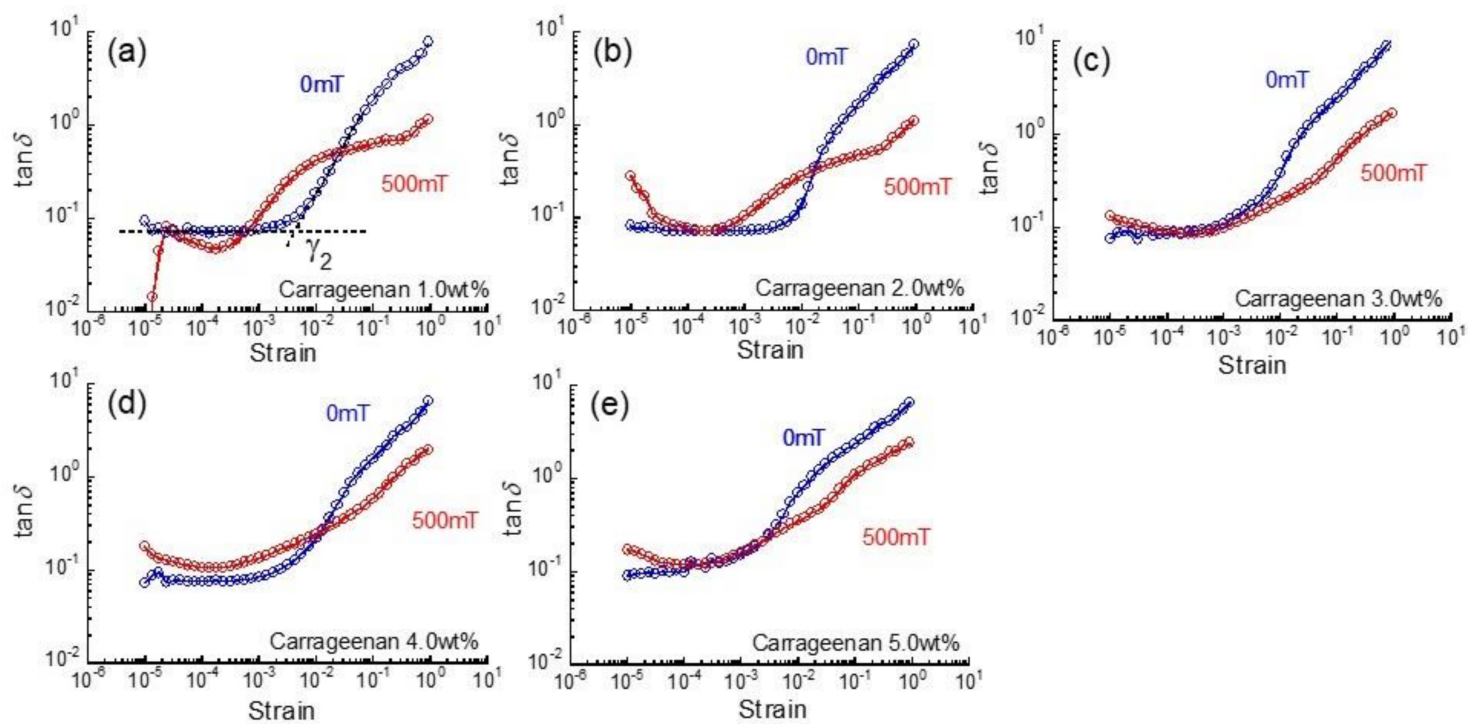

Figure 4. Strain dependence of loss tangent at 0 and $500 \mathrm{mT}$ for magnetic hydrogels with various carrageenan concentrations (CI: $70 \mathrm{wt} \%, f=1 \mathrm{~Hz}$ ), (a) $1.0 \mathrm{wt} \%$, (b) $2.0 \mathrm{wt} \%$, (c) $3.0 \mathrm{wt} \%$, (d) $4.0 \mathrm{wt} \%$, (e) $5.0 \mathrm{wt} \%$. 
The characteristic shear stresses $\sigma_{1}$ and $\sigma_{2}$ were explained by the following equations:

$$
\begin{aligned}
& \sigma_{1}=G^{\prime}{ }_{\gamma 1} \gamma_{1} \\
& \sigma_{2}=G^{\prime}{ }_{\gamma 2} \gamma_{2}
\end{aligned}
$$

where $G^{\prime}{ }_{11}$ and $G^{\prime}{ }_{\gamma 2}$ represent the storage moduli at $\gamma_{1}$ and $\gamma_{2}$, respectively. Figure 5a shows the relationship between characteristic stress $\sigma_{1}$ at $\gamma_{1}$ and the change in storage modulus for magnetic hydrogels. It was seen that the change in storage modulus decreased with the characteristic stress. This strongly suggests that magnetic particles are hard to move within the carrageenan network with the increasing of $\sigma_{1}$.
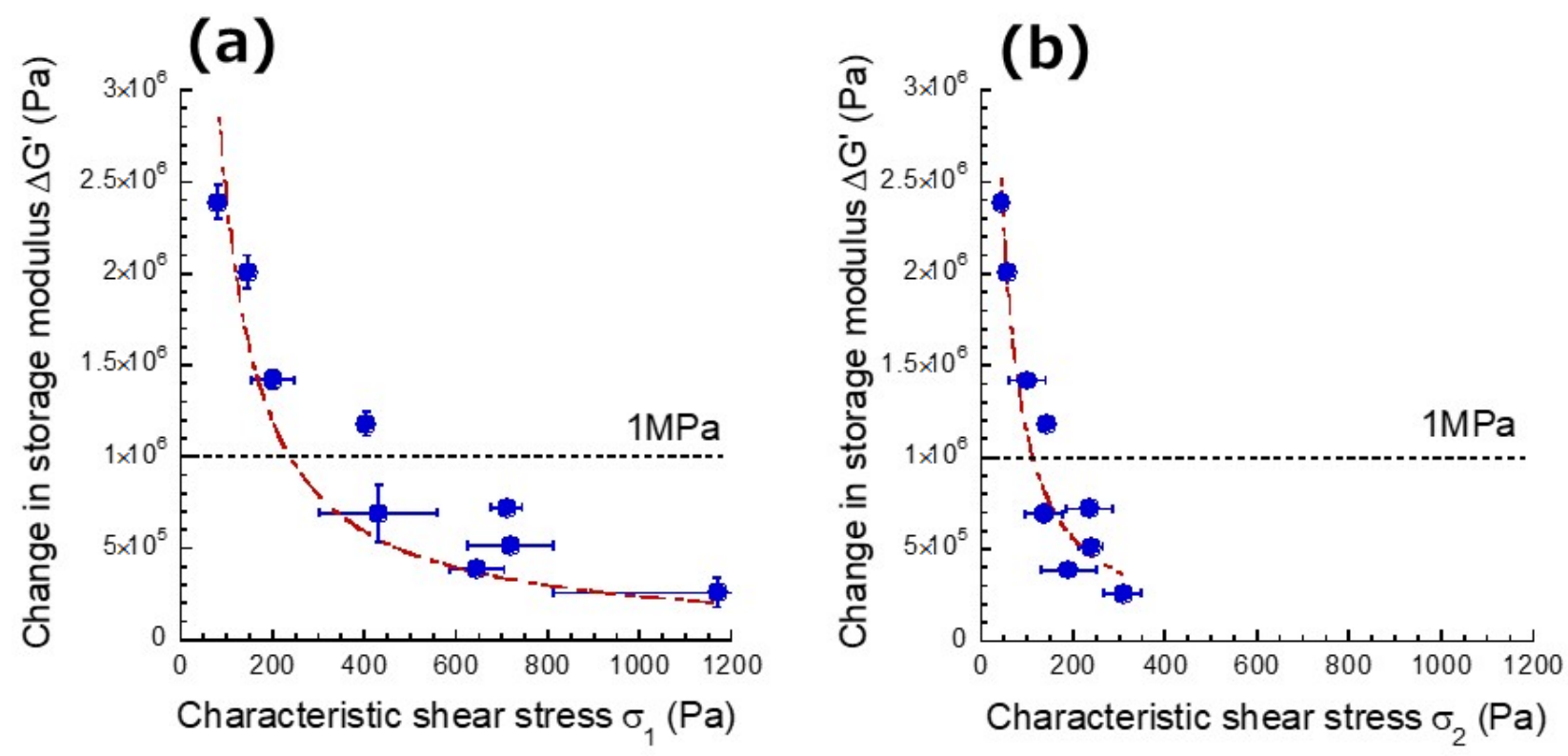

Figure 5. Relationship between change in storage modulus and characteristic stress (a) $\sigma_{1}$ or (b) $\sigma_{2}$ for magnetic hydrogels with various carrageenan concentrations (CI: $70 \mathrm{wt} \%)$.

Figure $5 \mathrm{~b}$ exhibits the relationship between characteristic stress $\sigma_{2}$ at $\gamma_{2}$ and the change in storage modulus for magnetic hydrogels. Similar to the behavior of $\sigma_{1}$, the change in storage modulus decreased with the characteristic stress, suggesting that the movement of magnetic particles is depressed with the increasing of $\sigma_{2}$. Thus, the characteristic stresses at $\gamma_{1}$ and $\gamma_{2}$ were distributed at 80-720 $\mathrm{Pa}$ and $40-310 \mathrm{~Pa}$, respectively. The dotted lines in the figures appear to show the change in storage modulus with $1 \mathrm{MPa}$. This means that a giant magnetorheology higher than $1 \mathrm{MPa}$ can be observed when the flowability of the gel-matrix is high, where the characteristic shear stresses satisfy a condition of $\tau_{1}<240 \mathrm{~Pa}$ or $\tau_{2}<110 \mathrm{~Pa}$.

Figure 6 shows the hysteresis in the strain dependence of storage modulus at 0 and $500 \mathrm{mT}$ for magnetic hydrogels and carrageenan hydrogels without magnetic particles. At $0 \mathrm{mT}$, there was no hysteresis in the storage modulus for both gels, regardless of the carrageenan concentration. As described in Figure 1, the carrageenan network was destructed by large strains higher than $10^{-3}-10^{-2}$. However, the destructed network perfectly recovered to the original modulus after the strain was reduced. At $500 \mathrm{mT}$, it was observed for both magnetic hydrogels $(1 \mathrm{wt} \%$ and $5 \mathrm{wt} \%$ carrageenan) that the storage modulus when reducing the strain was higher than that when increasing the strain. This behavior is typical, and has been observed for magnetic hydrogels, magnetic particles are highly aligned by both large strain and magnetic field. Our previous reports $[23,25]$ using a pulsatile magnetic field showed that the storage modulus for magnetic hydrogels reversibly changes in response to the magnetic field at approximately of 100 times. Accordingly, this 
strongly indicates that the storage modulus recovers to the original value although the magnetic particle does not recover to the original distribution.
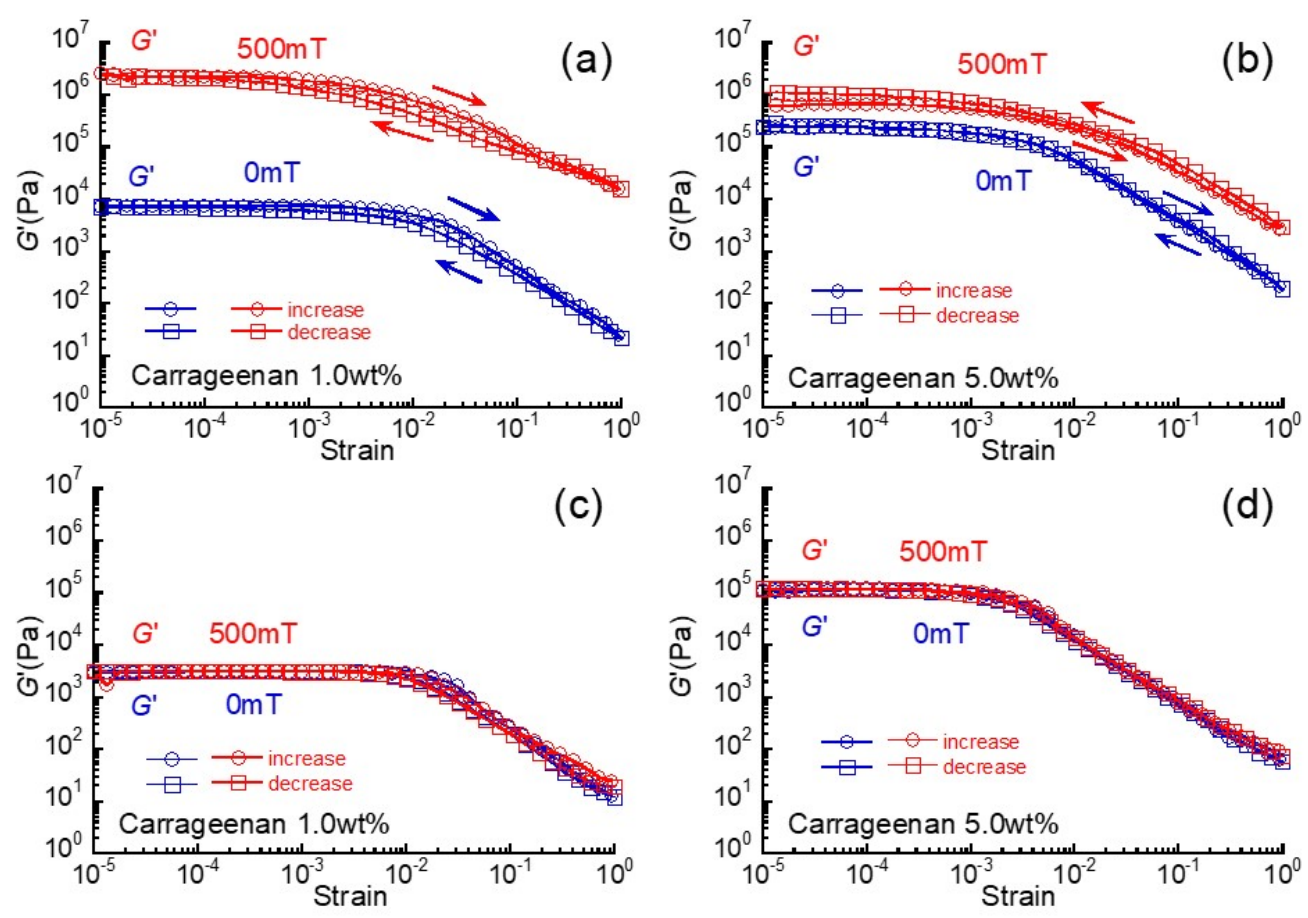

Figure 6. Hysteresis in the strain dependence of storage modulus for $(\mathbf{a}, \mathbf{b})$ magnetic hydrogels (CI: $70 \mathrm{wt} \%)$ and $(\mathbf{c}, \mathbf{d})$ carrageenan hydrogels without magnetic particles. $(\mathbf{a}, \mathbf{c}) 1 \mathrm{wt} \%$ carrageenan, $(\mathbf{b}, \mathbf{d})$ $5 \mathrm{wt} \%$ carrageenan.

Figure 7 indicates the SEM photographs for magnetic hydrogels taken at various experimental conditions in Figure 6. Note that all photographs were taken in the absence of a magnetic field. Figure 7a shows an image taken at initial state before applying large strains, which is the same as a magnetic hydrogel, as synthesized. Magnetic particles were randomly distributed in the matrix of carrageenan hydrogel, which agrees with our past studies [23,25]. Moreover, it is clear that magnetic particles were coated by carrageenan chains. It has been reported for fucan polysaccharide that there is a self-adhesion property between polysaccharides and magnetic particles [32]. Figure $7 \mathrm{~b}$ presents a photo taken after applying large strains, which corresponds to a condition after carrying out one-cycle strain dependence at $0 \mathrm{mT}$. Short fibers of carrageenan seem to be increased by large strains. Figure 7c shows a photo taken after applying a magnetic field of $500 \mathrm{mT}$ without applying shear. Figure $7 \mathrm{~d}$ exhibits a photo taken after applying large strains under the magnetic field. Many short fibers that connected magnetic particles are also seen in these images. The insets of Figure 7e-h show the SEM photographs for carrageenan hydrogels without magnetic particles. Thick fibers of carrageenan with several microns were observed in these images, which is typically observed in polysaccharide hydrogels. Generally, the thick fibers are rarely seen in magnetic hydrogels and the long filaments of carrageenan are observed as indicated by arrows in Figure $7 \mathrm{a}-\mathrm{d}$. There exists a hydrogen bond between -OH groups in carrageenan and on the surface of the CI particle [33]. By applying the magnetic field, the polysaccharide chains of carrageenan would be stretched by the relative movement of magnetic particles as the chain is partially adhered on the surface of CI particles. 

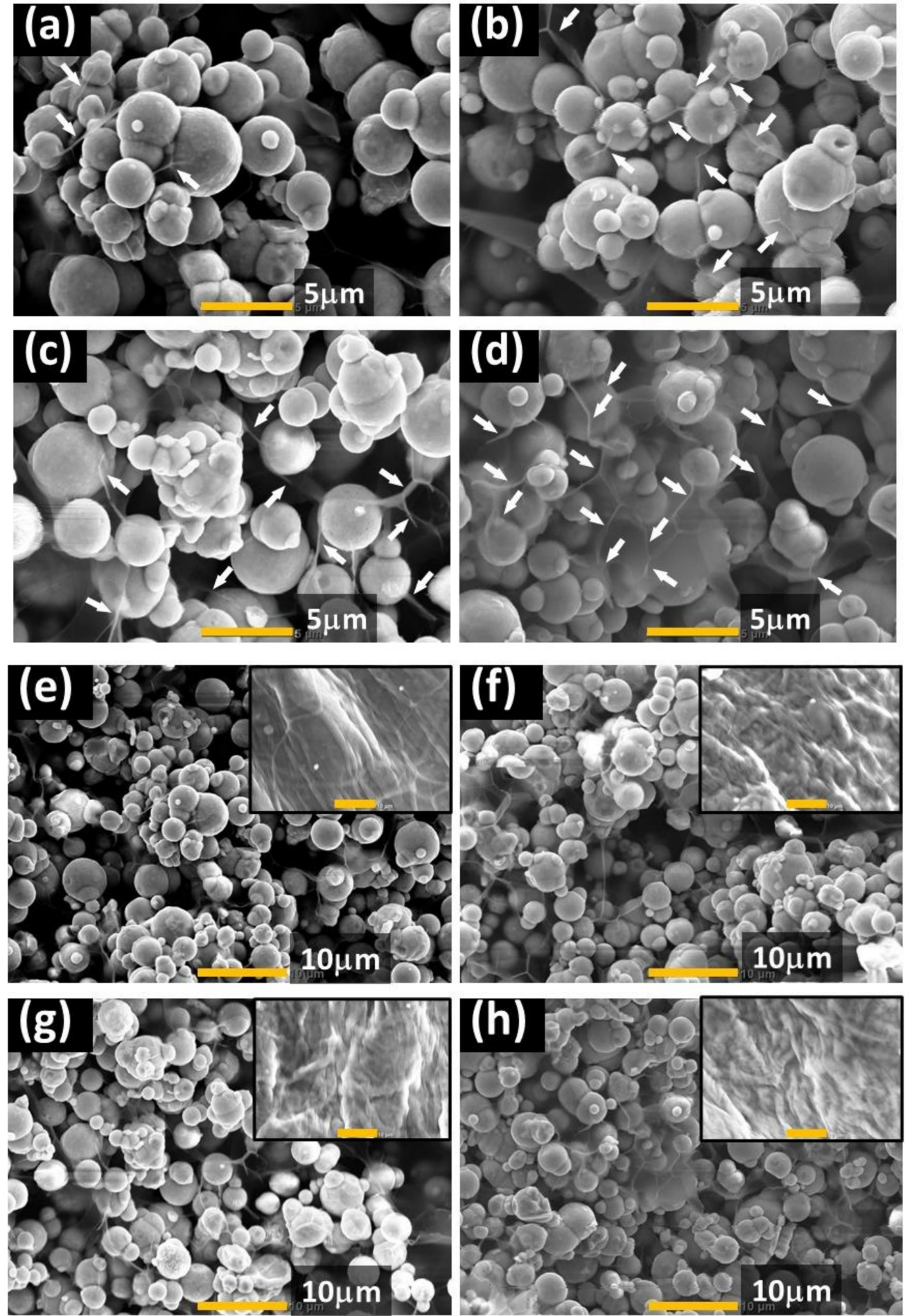

Figure 7. SEM photographs for $1 \mathrm{wt} \%$ carrageenan magnetic hydrogels taken at various conditions: $(\mathbf{a}, \mathbf{e})$ initial state before applying strain, $(\mathbf{b}, \mathbf{f})$ after applied large strain, $(\mathbf{c}, \mathbf{g})$ after applying a magnetic field of $500 \mathrm{mT}$, and $(\mathbf{d}, \mathbf{h})$ after applying large strain under a magnetic field of $500 \mathrm{mT}$. Inset: $1 \mathrm{wt} \%$ carrageenan hydrogels without magnetic particles. Magnification (a-d) $\times 5500$ (scale bar $=5 \mu \mathrm{m})$ and $(\mathbf{e}-\mathbf{h}) \times 2400$ (scale bar $=10 \mu \mathrm{m})$. Stretched fibers of carrageenan are indicated by arrows in $(\mathbf{a}-\mathbf{d})$.

\section{Conclusions}

The relationship between the flowability of the gel-matrix on the magnetorheological response was investigated using magnetic hydrogels with various carrageenan concentra- 
tions. The change in storage modulus decreased inversely proportional to the carrageenan concentration, suggesting that magnetic particles are difficult to move in the gel-matrix. As a parameter indicating the flowability of magnetic particles, we proposed two kinds of characteristic stresses $\sigma_{1}$ and $\sigma_{2}$. It was cleared that the change in storage modulus decreased inversely proportional to these characterisctic stresses. Additionaly, it was found that the stress $\sigma_{1}$ should be lower than 200-300 Pa to generate a modulus increase with an order of $1 \mathrm{MPa}$. SEM images revealed that the application of the magnetic field accelates fibering of carrageenan chains rather than the application of large strains. These insights could be useful for designing the polymer network of gels, enabling high mobility of magnetic particles.

\section{Materials and Methods}

\subsection{Synthesis of Magnetic Hydrogels}

The polymer matrix of magnetic hydrogels is $\mathrm{k}$-carrageenan of a polysaccharide $\left(M_{\mathrm{W}}=857 \mathrm{kDa}\right.$, CS-530, San-Ei Gen F.F.I., Osaka, Japan). Magnetic particles are carbonyl iron (CS Grade BASF SE., Ludwigshafen am Rhein, Germany). A pregel solution of the magnetic hydrogel was prepared by mixing $1 \mathrm{wt} \%$ carrageenan aqueous solution and magnetic particles at $100{ }^{\circ} \mathrm{C}$ using a vortex mixer for approximately $1 \mathrm{~min}$. The pregel solution was poured into a mold made of two sheets of glasses and a silicone spacer and was cooled to $20^{\circ} \mathrm{C}$ to obtain the magnetic hydrogel. The weight concentration of magnetic particles was kept at $70 \mathrm{wt} \%$, e.g., magnetic particle of $4.79 \mathrm{~g}$ and $1 \mathrm{wt} \%$ carrageenan aqueous solution of 2.05 g. Carbonyl iron (CI) particle (CS Grade BASF SE., Ludwigshafen am Rhein, Germany) was used as a magnetic particle, which has a saturation magnetization of $190 \mathrm{emu} / \mathrm{g}$ and a remanent magnetization of $1.6 \mathrm{emu} / \mathrm{g}$. The magnetization curve and the particle size distribution for the magnetic particle (same manufacturing lot number) were reported in previous papers $[24,26]$. The median diameter for magnetic particles was determined to be $7.0 \pm 0.3 \mu \mathrm{m}$ by a particle size analyzer (SALD-2200, Shimadzu Co. Ltd., Kyoto, Japan).

\subsection{Rheological Measurements}

The magnetic response of dynamic modulus for carrageenan hydrogels and magnetic hydrogels was measured by dynamic viscoelastic measurement using a rheometer (MCR301, Anton Paar Pty. Ltd., Graz, Austria) with an electromagnet system (PS-MRD) and a nonmagnetizable parallel plate (PP20/MRD) at $20^{\circ} \mathrm{C}$. The strain was varied from $10^{-5}$ to 1 , while the frequency was constant at $1 \mathrm{~Hz}$ at all measurements. The direction of shear is perpendicular to the magnetic field. The sample was a disk $20 \mathrm{~mm}$ in diameter and $1.5 \mathrm{~mm}$ in thickness. The data of storage modulus shown in Figures 2, 3 and 5 were determined by averaging over three different samples in a single batch.

\subsection{SEM Observations}

Scanning electron microscope (SEM) observation was carried out using JCM-6000 Neoscope (JEOL Ltd. Tokyo, Japan) with an accelerating voltage of $15 \mathrm{kV}$ without Au coating. Magnetic hydrogels with a size of $\varphi 20 \mathrm{~mm} \times \mathrm{t} 1 \mathrm{~mm}$ were dried by freeze-drying for $5 \mathrm{~h}$ without applying a magnetic field. The cross section of these dried gels was observed. Apparent shrinkage was not observed for the magnetic gel owing to the freeze-drying (less than $3 \%$ in length).

Author Contributions: The idea for this work comes from J.I. and M.K. J.I. wrote the paper. M.K. conceived and designed the experiments. J.I., T.K. and K.O. performed sample preparation, particle diameter analysis, rheological experiment, and microscope observation. S.A. carried out preexperiment. Conceptualization and supervision were made by T.M. Presentation of the results and the draft structure were discussed with all authors. All authors have read and agreed to the published version of the manuscript. 
Funding: This research was partially supported by a grant from the Cooperative Research Program of the Network Joint Research Center for Materials and Devices (number 20201324), Nagai NS Promotion Foundation for Science of Perception.

Data Availability Statement: The data presented in this study are available in article.

Acknowledgments: The authors are grateful to San-Ei Gen F.F.I., Inc. for the offer of sample.

Conflicts of Interest: The authors declare that there is no conflict of interest.

\section{References}

1. Kalita, V.M.; Dzhezherya, Y.I.; Cherepov, S.V.; Skirta, Y.B.; Bodnaruk, A.V.; Levchenko, G.G. Critical bending and shape memory effect in magnetoactive elastomers. Smart Mater. Struct. 2021, 30, 025020. [CrossRef]

2. Alekhina, Y.A.; Makarova, L.A.; Kostrov, S.A.; Stepanov, G.V.; Kazimirova, E.G.; Perov, N.S.; Kramarenko, E.Y. Development of magnetoactive elastomers for sealing eye retina detachments. J. Appl. Polym. Sci. 2019, 136, 47425. [CrossRef]

3. Kang, S.S.; Choi, K.; Nam, J.D.; Choi, H.J. Magnetorheological Elastomers: Fabrication, Characteristics, and Applications. Materials 2020, 13, 4597. [CrossRef] [PubMed]

4. Kalita, V.M.; Dzhezherya, Y.I.; Levchenko, G.G. Anomalous magnetorheological effect in unstructured magnetoisotropic magnetoactive elastomers. Appl. Phys. Lett. 2020, 116, 063701. [CrossRef]

5. Khayama, S.U.; Muhammad, U.; Malik, A.U.; Ahmed, R. Development and characterization of a novel hybridmagnetorheological elastomer incorporating micro and nano size iron fillers. Mater. Des. 2020, 192, 108748. [CrossRef]

6. Norhiwani, M.H.; Saiful, A.M.; Ubaidillah, U.; Siti, A.A.A.; Muntaz, H.A.K.; Nur, A.N.; Nazmi, N. Solvent Dependence of the Rheological Properties in Hydrogel Magnetorheological Plastomer. Int. J. Mol. Sci. 2020, 21, 1793.

7. Fischer, L.; Menzel, A.M. Magnetically induced elastic deformations in model systems of magnetic gels and elastomers containing particles of mixed size. Smart Mater. Struct. 2021, 30, 014003. [CrossRef]

8. Fischer, L.; Menzel, A.M. Magnetostriction in magnetic gels and elastomers as a function of the internal structure and particle distribution. J. Chem. Phys. 2019, 151, 114906. [CrossRef]

9. Qianqian, W.; Yu, W.; Jiabin, F.; Xinglong, G. Transient response of magnetorheological elastomers to step magnetic field. Appl. Phys. Lett. 2018, 113, 081902.

10. Szabo, D.; Szeghy, G.; Zrinyi, M. Shape Transition of Magnetic Field Sensitive Polymer Gels. Macromolecules 1998, 31, 6541-6548. [CrossRef]

11. Stepanov, G.V.; Abramchuk, S.S.; Grishin, D.A.; Nikitin, L.V.; Kramarenko, E.Y.; Khokhlov, A.R. Effect of a homogeneous magnetic field on the viscoelastic behavior of magnetic elastomers. Polymer 2007, 48, 488-495. [CrossRef]

12. Gundermann, T.; Odenbach, S. Investigation of the motion of particles in magnetorheological elastomers by X- $\mu$ CT. Smart Mater. Struct. 2014, 23, 105013. [CrossRef]

13. Watanabe, M.; Takeda, Y.; Maruyama, T.; Ikeda, J.; Kawai, M.; Mitsumata, T. Chain Structure in a Cross-Linked Polyurethane Magnetic Elastomer Under a Magnetic Field. Int. J. Mol. Sci. 2019, 20, 2879. [CrossRef]

14. Mitsumata, T.; Honda, A.; Kanazawa, H.; Kawai, M. Magnetically Tunable Elasticity for Magnetic Hydrogels Consisting of Carrageenan and Carbonyl Iron Particles. J. Phys. Chem. B 2012, 116, 12341-12348. [CrossRef]

15. Wu, Z.; Deng, W.; Zhou, W.; Luo, J. Novel magnetic polysaccharide/grapheme oxide @ $\mathrm{Fe}_{3} \mathrm{O}_{4}$ gel beads for adsorbing heavy metal ions. Carbohydr. Polym. 2019, 216, 119-128. [CrossRef]

16. Daniel-da-silva, A.L.; Moreira, J.; Neto, R.; Estrada, A.C.; Gil, A.M.; Trindade, T. Impact of magnetic nanofillers in the swelling and release properties of $\mathrm{K}$-carrageenan hydrogel nanocomposites. Carbohydr. Polym. 2012, 87, 328-335. [CrossRef]

17. Sagbas, S.; Butun, S.; Sahiner, N. Modifiable chemically crosslinked poli (k-carrageenan) particles. Carbohydr. Polym. 2012, 87, 2718-2724. [CrossRef]

18. Duman, O.; Tunç, S.; Polat, T.G.; Bozo $`$ glan, B.K. Synthesis of magnetic oxidized multiwalled carbon nanotube-k-carrageenan$\mathrm{Fe}_{3} \mathrm{O}_{4}$ nanocomposite adsorbent and its application in cationic Methylene Blue dye adsorption. Carbohydr. Polym. 2016, 147, 79-88. [CrossRef]

19. Chomoucka, J.; Drbohlavova, J.; Huska, D.; Adam, V.; Kizek, R.; Hubalek, J. Magnetic nanoparticles and targeted drug delivering. Pharmacol. Res. 2010, 62, 144-149. [CrossRef]

20. Katagiri, K.; Ohta, K.; Sako, K.; Inumaru, K.; Hayashi, K.; Sasaki, Y.; Akiyoshi, K. Development and Potential Theranostic Applications of a Self-Assembled Hybrid of Magnetic Nanoparticle Clusters with Polysaccharide Nanogels. Chem. Plus Chem. 2014, 79, 1631-1637. [CrossRef]

21. Wang, C.; Gao, X.; Chen, Z.; Chen, Y.; Chen, H. Preparation, Characterization and Application of Polysaccharide-Based Metallic Nanoparticles. Polymers 2017, 9, 689. [CrossRef]

22. Balachandramohana, J.; Anandanb, S.; Sivasankara, T. A simple approach for the sonochemical synthesis of $\mathrm{Fe}_{3} \mathrm{O}_{4}$-guargum nanocomposite and its catalytic reduction of $p$-nitroaniline. Ultrason. Sonochem. 2018, 40, 1-10. [CrossRef]

23. Mitsumata, T.; Abe, N. Magnetic-field Sensitive Gels with Wide Modulation of Dynamic Modulus. Chem. Lett. 2009, 38, 922-923. [CrossRef] 
24. Akama, S.; Ikeda, J.; Kawai, M.; Mitsumata, T. A Feature in Magnetorheological Effect for Polysaccharide Magnetic Hydrogels. Chem. Lett. 2018, 47, 1240-1242. [CrossRef]

25. Kimura, Y.; Kanauchi, S.; Kawai, M.; Mitsumata, T.; Tamesue, S.; Yamauchi, T. Effect of Plasticizer on the Magnetorheological Response for Magnetic Polyurethane Elastomers. Chem. Lett. 2015, 44, 177-178. [CrossRef]

26. Ikeda, J.; Takahashi, D.; Watanabe, M.; Kawai, M.; Mitsumata, T. Particle Size in Secondary Particle and Mangetic Response for Carrageenan magnetic Hydrogels. Gels 2019, 5, 39. [CrossRef] [PubMed]

27. Matricardi, P.; Alhaique, F.; Coviello, T. Polysaccharide Hydrogels, Characterization and Biomedical Applications; Jenny Stanford Publishing: New York, NY, USA, 2015.

28. Sijun, L.; Lin, L. Recoverable and Self-Healing Double Network Hydrogel Based on k-Carrageenan. ACS Appl. Mater. Interfaces 2016, 8, 29749-29758.

29. Sijun, L.; Lin, L. Ultrastretchable and Self-Healing Double-Network Hydrogel for 3D Printing and Strain Sensor. ACS Appl. Mater. Interfaces 2017, 9, 26429-26437.

30. Sijun, L.; Hongbin, Z.; Wei, Y. Simultaneously improved strength and toughness in K-carrageenan/polyacrylamide double network hydrogel via synergistic interaction. Carbohydr. Polym. 2020, 230, 115596.

31. Kyu, H.; Sook, H.K.; Kyung, H.A.; Seung, J.L. Large amplitude oscillatory shear as a way to classify the complex fluids J. Non-Newton. Fluid Mech. 2002, 107, 51-65.

32. Silva, V.A.J.; Andrade, P.L.; Silva, M.P.C.; Bustamante, A.; Valladares, L.D.L.S.; Aguiar, J.A. Synthesis and characterization of $\mathrm{Fe}_{3} \mathrm{O}_{4}$ nanoparticles coated with fucan polysaccharides. J. Magn. Magn. Mater. 2013, 343, 138-143. [CrossRef]

33. Takahashi, D.; Watanabe, M.; Kawai, M.; Mitsumata, T. Magnetorheological Response for Magnetic Elastomers Containing Carbonyl Iron Particles Coated with Poly(methyl methacrylate). Polymers 2021, 13, 335. [CrossRef] [PubMed] 